\title{
MiR-34b/c rs4938723 Polymorphism Significantly Decreases the Risk of Digestive Tract Cancer: Meta-analysis
}

\author{
Tian-Xing Ji ${ }^{1 \& *}$, Cheng Zhi $^{2 \&}$, Xue-Guang Guo ${ }^{3}$, Qiang Zhou ${ }^{1}$, Guo-Qiang Wang ${ }^{4}$, \\ Bo Chen ${ }^{1}$, Fei-Fei Ma ${ }^{5 *}$
}

\begin{abstract}
Background: Previous studies investigating the association between miR-34b/c rs4938723 polymorphism and cancer risk showed inconclusive. Here, we performed meta-analysis to investigate the association between miR34b/c rs4938723 polymorphism and digestive cancer risk. Materials and Methods: Literature database including PubMed, OVID, Chinese National Knowledge Infrastructure (CNKI) were searched for publications concerning the association between the miR-34b/c rs4938723 polymorphism and digestive cancer risk. Results: A total of 6 studies consisting of 3246 cases and 3568 controls were included in this meta-analysis. The combined analysis suggested the miR-34b/c rs4938723 polymorphism significantly reduced digestive cancer risk under allelic model, homogeneous co-dominant model and recessive model $(\mathrm{C}$ vs $\mathrm{T}$ : $\mathrm{OR}=\mathbf{0 . 8 8 , 9 5 \%} \mathrm{CI}=\mathbf{0 . 8 2 - 0 . 9 5}, \boldsymbol{p}$-value $=0.001 ; \mathrm{CC}$ vs TT: $\mathrm{OR}=0.67,95 \% \mathrm{CI}=0.57-0.80, \boldsymbol{p}$-value $=0.000 ; \mathrm{CC}$ v $s$ TT/TC: $\mathrm{OR}=\mathbf{0 . 6 8 , 9 5 \%} \mathrm{CI}=\mathbf{0 . 5 8 - 0 . 8 0 ,} \boldsymbol{p}$-value $=\mathbf{0 . 0 0 0}$ ). Q-test and 12 test revealed no significant heterogeneity in all genotype comparisons. The Begger's funnel plot and Egger's test did not show significant publication bias. Conclusions: The current evidence supports the conclusion that the miR-34b/c rs4938723 polymorphism decreases an individual's susceptibility to digestive cancers.
\end{abstract}

Keywords: miR-34b/c - polymorphism - digestive cancer - susceptibility

Asian Pac J Cancer Prev, 16 (14), 6099-6104

\section{Introduction}

$\mathrm{MiR}-34 \mathrm{~b} / \mathrm{c}$ is member of tumor suppressor miR-34 family, which is a downstream transcriptional target of p53 (Corney et al., 2007; He et al., 2007). Accumulating reports have demonstrated that miR-34 b/c was down-regulated in multiple types of cancer mainly via hyper-methylation. It was proposed hyper-methylation of miR-34b/c CpG island is biomarker of multiple cancer and is linked to cancer progression and prognosis suggesting deregulation of miR-34b/c involve in p53-muted cancer and p53 normal cancer (Lujambio et al., 2008; Kalimutho et al., 2011; Vogt et al., 2011; Chen et al., 2012; Suzuki et al., 2014; Wang et al., 2014). Recent studies found $\mathrm{miR}-34 \mathrm{~b} / \mathrm{c}$ single nucleotide polymorphisms (SNPs) rs4938723 locating in a typical $\mathrm{CpG}$ islands affected predicted GATA-X transcription factors binding and miR-34b/c expression, ultimately altered cancer susceptibility (Xu et al., 2011; Son et al., 2013). However, studies and meta-analyses on $\mathrm{miR}-34 \mathrm{~b} / \mathrm{c}$ rs4938723 polymorphism and cancer risks shown inconclusive results (Bensen et al., 2013; Gao et al., 2013; Han et al., 2013; Li et al., 2013; Yin et al., 2013; Oh et al., 2014; Tian et al., 2014; Yang et al., 2014; Zhang et al., 2014a; Zhang et al., 2014b; Pan et al., 2015). In addition, the association between miR-34b/c rs4938723 polymorphism and digestive cancer had not been assessed (Tao et al., 2014; Yi et al., 2014). Therefore, we performed a meta-analysis on all eligible case-control studies to estimate effect of miR-34b/c rs4938723 polymorphism on the digestive cancer risk.

\section{Materials and Methods}

\section{Literature search}

In order to identify the relevant papers about miR$34 \mathrm{~b} / \mathrm{c}$ rs4938723 polymorphism and digestive cancer risk, we performed a systematic search from PubMed, OVID, Chinese National Knowledge Infrastructure (CNKI) databases, with a combination of the following keywords: " miR-34b/c, microRNA-34b/c, pre-miR-34b/c" ; "rs4938723"; "allele mutation or polymorphism" (last search was updated on 2 Mar. 2015). References of previous meta-analyses and reviews were also manually searched to identify additional studies. We evaluated potentially relevant publications by examining their titles and abstracts and all studies matching the eligible criteria were retrieved.

${ }^{1}$ Department of Clinical Laboratory, ${ }^{2}$ Department of Clinical Pathology, ${ }^{4}$ Department of Gastrointestinal Surgery, Lab of Surgery, ${ }^{5}$ Department of VIP Obstetrics, the Second Affiliated Hospital of Guangzhou Medical University, ${ }^{3}$ Department of Clinical Laboratory, the Third Affiliated Hospital of Guangzhou Medical University, Guangzhou, China ${ }^{\circledR}$ Equal contributors *For correspondence: dream125@126.com, jitianxing7021@163.com 
Table 2. Meta-analysis of the miR-34b/c (rs4938723) Polymorphism and Digestive Cancer Risk under all Genotype Model

\begin{tabular}{|c|c|c|c|c|c|c|c|}
\hline \multirow{2}{*}{$\begin{array}{l}\text { Total } \\
\text { analysis }\end{array}$} & \multirow{2}{*}{$\begin{array}{c}\text { Analysis } \\
\text { model }\end{array}$} & \multicolumn{2}{|c|}{ Test of association } & \multicolumn{2}{|c|}{ Test for heterogeneity } & \multirow{2}{*}{$\begin{array}{l}\text { Begg's } \\
\text { Test } \\
p \text {-value }\end{array}$} & \multirow{2}{*}{$\begin{array}{c}\text { Egger's } \\
\text { test } \\
p \text {-value }\end{array}$} \\
\hline & & OR $(95 \%$ CI $)$ & $p$-value & $p$-value & $\mathrm{I}^{2}$ & & \\
\hline $\mathrm{C} v s \mathrm{~T}$ & $\mathrm{~F}$ & 0.88 [0.82- 0.95$]$ & $0.001 *$ & 0.219 & $28.80 \%$ & 0.26 & 0.106 \\
\hline $\mathrm{TC} v s \mathrm{TT}$ & $\mathrm{F}$ & 0.98 [0.89- 1.09] & 0.731 & 0.102 & $45.50 \%$ & $0.009 *$ & $0.029 *$ \\
\hline $\mathrm{CC} v s \mathrm{TT}$ & $\mathrm{F}$ & $0.67[0.57-0.80]$ & $0.000 *$ & 0.768 & $0.00 \%$ & 0.707 & 0.548 \\
\hline $\mathrm{CC}$ ss TT/TC & $\mathrm{F}$ & $0.68[0.58-0.80]$ & $0.000 *$ & 0.877 & $0.00 \%$ & 0.707 & 0.731 \\
\hline $\mathrm{CC} / \mathrm{TC} v s \mathrm{TT}$ & $\mathrm{F}$ & $0.92[0.84-1.01]$ & 0.088 & 0.097 & $46.30 \%$ & $0.024 *$ & $0.039 *$ \\
\hline $\mathrm{TC} v s \mathrm{TT} / \mathrm{CC}$ & $\mathrm{F}$ & $1.05[0.96-1.16]$ & 0.303 & 0.165 & $36.30 \%$ & $0.009 *$ & $0.029 *$ \\
\hline
\end{tabular}

\section{Inclusion and exclusion criteria}

Studies included in the current meta-analysis must conform to all the following criteria: (a) evaluation of miR-34b/c rs4938723 and digestive cancer risks, (b) use a case-control design, (c) sufficient published data for estimating an odds ratio (OR) with $95 \%$ confidence interval (CI).

The exclusion criteria were as follows: (a) study pertaining to other SNP of miR34b/c and cancer risk, but not rs4938723; (b) review or meta-analysis concerning $\mathrm{miR}-34 \mathrm{~b} / \mathrm{c}$ rs4938723 and cancer risks. (c) Study on miR-34b/c rs4938723 and non-digestive cancer risk.

\section{Quality assessment}

The quality of each study was assessed according to the quality assessment criteria recommended by Xue et al (Supplemental Table 1). Quality scores of studies ranged from 0 (lowest) to 15 (highest). Studies with score less than 9 were considered as low quality, while those scores equal to or greater than 9 were categorized into high quality (Xue et al., 2015).

\section{Data extraction}

Two investigators independently extracted the data, finally reached consensus on all items. For each study, the following parameters were extracted: the first author's last name, year of publication, country of origin, ethnicity, the numbers of genotyped cases and controls and genotyping methods.

\section{Statistical analysis}

OR corresponding to $95 \% \mathrm{CI}$ was used to assess the strength of association between rs4938723 polymorphism and digestive cancer risk. The significance of the pooled OR was determined by the Z-test, and $p$-value 0.05 was considered as statistically significant. We explored the association between rs4938723 and digestive cancer risk using allelic model ( $\mathrm{C} v s \mathrm{~T}$ ), co-dominant model (homogeneous co-dominant model: CC vs TT, heterogeneous co-dominant model: TC vs TT), recessive $(\mathrm{CC} v s \mathrm{TC}+\mathrm{TT})$, over-dominant $(\mathrm{TC} v s \mathrm{TT}+\mathrm{CC})$ and dominant $(\mathrm{CC}+$ TC vs TT) genetic models. A chi-square-based Q-statistic test and an I2-test test were both carried out to evaluate the heterogeneity of the studies. By heterogeneity test, if $p$-value 0.10 for the Q-test, the pooled OR was calculated by the fixed effects model (Mantel and Haenszel, 1959). Otherwise, the random-effects model (DerSimonian and Laird, 1986). Hardy-Weinberg equilibrium (HWE) in the control group was estimated using Fisher's exact test and a $p$-value 0.05 was considered significant. Leave-one-study-out sensitivity analysis was performed to reflect the influence of the individual data-set to the pooled OR (Normand, 1999). Publication bias was evaluated using the funnel plot and Egger's test (Egger et al., 1997). $p$-value0.05 indicate the presence of potential publication bias. All statistical tests were performed with Stata ES 12.0 (Stata Corporation, College Station, TX, USA).

\section{Results}

\section{Study characteristics}

The Processes of studies searching and selecting are illustrated in the flow diagram (Figure 1). A total of 204 articles were achieved by literature search, 
from PubMed, OVID, Chinese National Knowledge Infrastructure (CNKI) databases, using different combination of key terms. 11 articles were obtained by browsing the reference of meta-analysis or articles pertaining to $\mathrm{miR}-34 \mathrm{~b} / \mathrm{c}$ rs4938723 polymorphism and cancer risk. 47 records were excluded for duplicate, 144 records were excluded improper titles and/or abstracts. Then, 24 eligible studies were retrieved for detailed evaluation. During the extraction of data, 18 articles were excluded (ten were meta-analysis, one study evaluated mir-34b rs2187473 (T/C) polymorphisms and oral carcinoma risk, but not miR-34b/c rs4938723, 7 studies evaluated the relationship between mir-34b rs2187473 (T/C) polymorphisms and non-digestive cancer). As a result, a total of 6 publications including 3246 cases and 3568 controls were included in the meta-analysis [16-21].

The characteristics of the included were summarized in Table 1. In the eligible studies, there were 2 studies

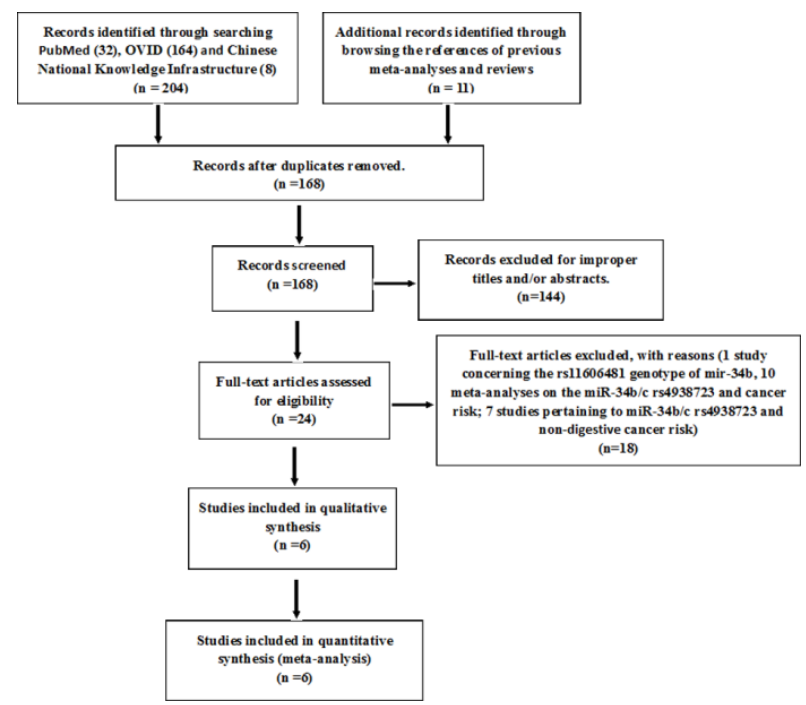

Figure 1. Flow Diagram of Study Identification of esophageal cancer, 2 studies of gastric cancer and 2 studies of colorectal cancer. 5 studies come from china, 1 study from Korea. The controls of 5 studies came from hospital-based gender and age matched healthy population; the gender of control did not matched to that of cases in one study. The distribution of genotypes in the controls in all eligible studies did not deviate from HWE (Table 1). Besides, each study included in the meta-analysis was regarded as high quality according to the quality assessment criteria recommended by xue et al (Supplemental Table 1) (Xue et al., 2015).

\section{Meta-analysis results}

In overall population, there was significant heterogeneity in miR-34b/c rs4938723 for all model comparison including allelic model, homogeneous codominant model, heterogeneous co-dominant model, recessive model comparisons and dominant model comparison, and over-dominant model (Table 2 and Figure 1). The pooled meta-analysis suggested that rs4938723 was associated with lower digestive cancer risk under allelic model, homogeneous co-dominant model and recessive model $(\mathrm{C} v s \mathrm{~T}: \mathrm{OR}=0.88,95 \% \mathrm{CI}=0.82-0.95$, $p$-value $=0.001 ; \mathrm{CC}$ ss TT: OR $=0.67,95 \% \mathrm{CI}=0.57-$ $0.80, p$-value $=0.000 ; \mathrm{CC}$ vs TT/TC: $\mathrm{OR}=0.68,95 \% \mathrm{CI}$ $=0.58-0.80, p$-value $=0.000)$ (Table 2, Figure 2 and 4). However, we did not found the association between rs4938723 polymorphism and digestive cancer risk in overall population under heterogeneous co-dominant model, dominant model comparison and over-dominant model (Table 2). Meanwhile, there was no significant heterogeneity in all genetic models comparison (Table 2 and Figure 1).

\section{Publication bias}

Begger's funnel plot and Egger's test were performed

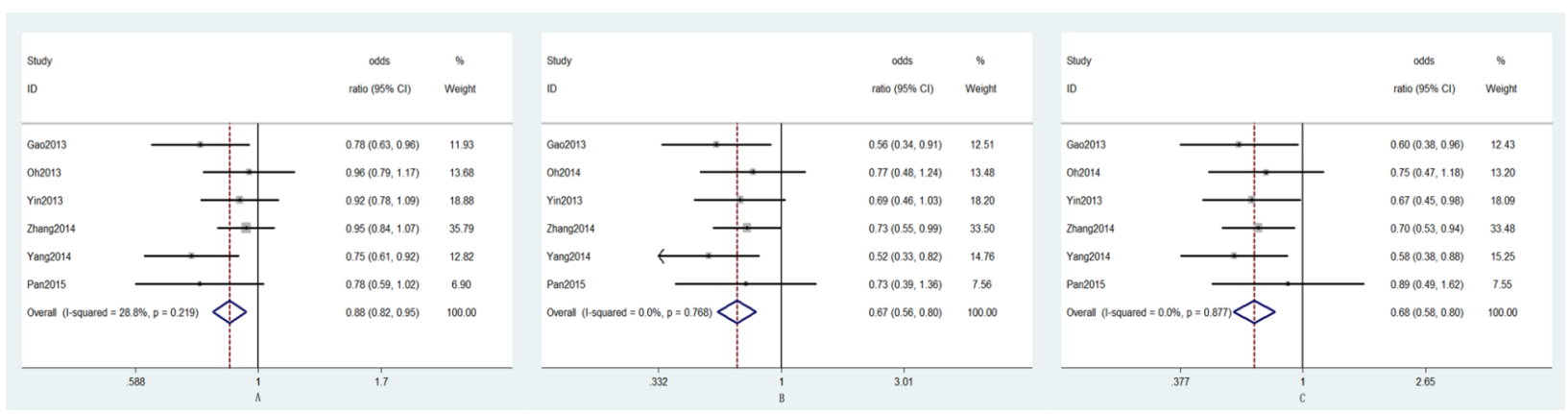

Figure 2. Forest Plot of miR-34b/c rs4938723 Polymorphism and Risk of Digestive Cancer. (A). C vs T; (B) CC vs TT; (C) CC vs TT+TC
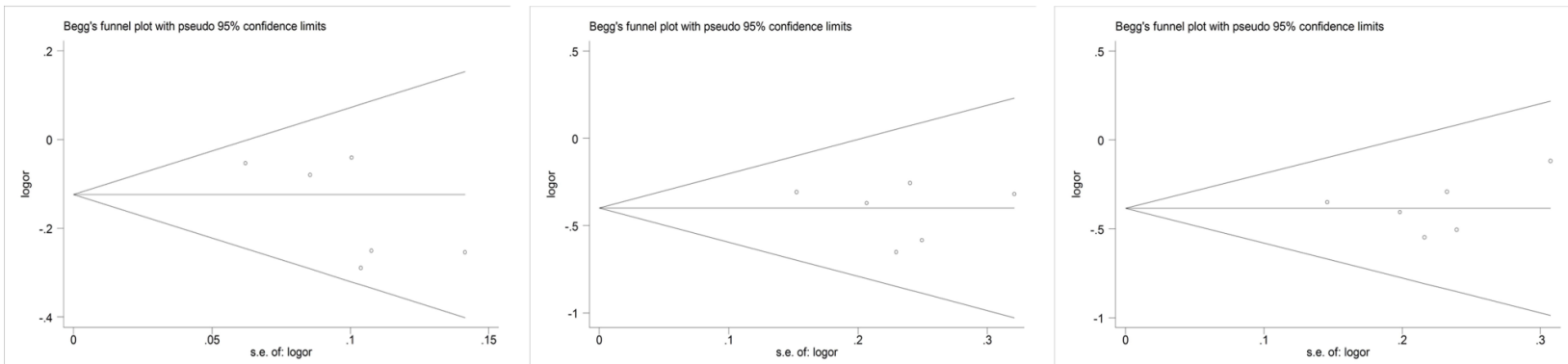

Figure 3. Begg's Funnel Plot for Publication Bias Test. (A). C vs T; (B) CC vs TT; (C) CC vs TT+TC 


\begin{tabular}{|c|c|c|c|c|c|c|c|}
\hline Criteria and Score & & Gao, 2013 & Oh, 2014 & Yin, 2013 & Zhang, 2014 & Yang, 2014 & Pan, 2015 \\
\hline \multicolumn{8}{|l|}{ Representativeness of case } \\
\hline Selected from population cancer registry & 2 & & & & & & \\
\hline Selected from hospital & 1 & 1 & 1 & 1 & 1 & 1 & 1 \\
\hline No method of selection described & 0 & & & & & & \\
\hline \multicolumn{8}{|l|}{ Representativeness of control } \\
\hline Population-based & 3 & & & & 3 & & \\
\hline Mixed & 2 & & & & & & \\
\hline Hospital-based & 1 & 1 & 1 & 1 & & 1 & 1 \\
\hline Not described & 0 & & & & & & \\
\hline \multicolumn{8}{|l|}{ Ascertainment of cancer case } \\
\hline Histopathologic confirmation & 2 & 2 & 2 & 2 & 2 & 2 & 2 \\
\hline by patient medical record & 1 & & & & & & \\
\hline Not described & 0 & & & & & & \\
\hline \multicolumn{8}{|l|}{ Control selection } \\
\hline Controls matched with cases by age and sex & 2 & 2 & & 2 & 2 & 2 & 2 \\
\hline Controls matched with cases only by age or by sex & 1 & & 1 & & & & \\
\hline Not matched or not described & 0 & & & & & & \\
\hline \multicolumn{8}{|l|}{ Genotyping examination } \\
\hline Genotyping done blindly and quality control & 2 & 2 & & & & & \\
\hline Only genotyping done blindly or quality control & 1 & & 1 & 1 & 1 & 1 & 1 \\
\hline Not described & 0 & & & & & & \\
\hline \multicolumn{8}{|l|}{ HWE } \\
\hline HWE in the control group & 1 & 1 & 1 & 1 & 1 & 1 & 1 \\
\hline HWD in the control group or not mentioned & 0 & & & & & & \\
\hline \multicolumn{8}{|l|}{ Total sample size } \\
\hline$>1000$ & 3 & & & 3 & 3 & & \\
\hline $501-1000$ & 2 & 2 & 2 & & & 2 & \\
\hline $201-500$ & 1 & & & & & & 1 \\
\hline$\leq 200$ & 0 & & & & & & \\
\hline Sum & & 11 & 9 & 11 & 13 & 10 & 9 \\
\hline
\end{tabular}

to assess the publication bias of included studies. As shown in Figure 3, the shapes of the funnel plots reveal obvious symmetry under allelic model, homogeneous co-dominant model and recessive model comparison. Then, Egger's test was used to provide further statistical evidence of funnel plot symmetry (Table 2). However, there were publication bias under heterogeneous co-dominant model, dominant model comparison and over-dominant model comparison which were not found to be related to the susceptibility of digestive cancer (Table 2).

\section{Sensitivity analysis}

We deleted one single study from the overall pooled analysis each time to check the influence of the removed data set to the overall ORs. The pooled ORs and 95\% CIs were not significantly altered when any part of the study was omitted, which indicated that any single study had little impact on the overall ORs (data not shown).

\section{Discussion}

In current study, meta-analysis between $\mathrm{miR}-34 \mathrm{~b} / \mathrm{c}$ rs4938723 and digestive cancer risk was performed. There was not significant heterogeneity under all genetic models (Table 2). And the pooled analysis indicted the variant $\mathrm{C}$ allele and $\mathrm{CC}$ homozygote was associated with a lower digestive cancer risk compared to the T allele, TT wildtype homozygote and TC/CC genotype. However, recent studies and meta-analysis suggested the $\mathrm{T}$ to $\mathrm{C}$ shift of the rs4938723 polymorphism could increase certain cancer risk including hepatocellular, renal and nasopharyngeal carcinoma; was not linked to certain cancer susceptibility such as breast cancer (Xu et al., 2011; Li et al., 2013; Wang et al., 2013; Yi et al., 2014; Zhang et al., 2014b). Moreover, Zhang et al (Zhang et al., 2014b) further experimentally ascertained the expression of miR-34b/c was higher in in normal renal tissues with TT+TC genotypes than in those with CC genotypes, the luciferase activities with rs4938723 T allele in 293-T cells was higher than that with $\mathrm{C}$ allele. Therefore, $\mathrm{C}$ allele of rs 4938723 polymorphism has different effects on different cancer types by affect the expression of $\mathrm{miR}-34 \mathrm{~b} / \mathrm{c}$.

It was proposed that mechanisms involving in the down-regulation of $\mathrm{miR}-34 \mathrm{~b} / \mathrm{c}$ included inactivating mutations of $\mathrm{p} 53$, hyper-methylation and mutation of its encoding genes (Lujambio et al., 2008; Toyota et al., 2008; Corney et al., 2010; Suzuki et al., 2010). MiR-34b/c SNP rs4938723 locates in a typical CpG island, may affect predicted GATA-X transcription factors binding and methylation status of $\mathrm{miR}-34 \mathrm{~b} / \mathrm{c} \mathrm{CpG}$ islands. It is plausible that the miR-34b/c polymorphism rs4938723 might affect cancer susceptibility by creating the predicted GATA-binding site or the $\mathrm{CpG}$ methylation (Gao et al., 2013; Zhang et al., 2014b). Furthermore, other cancer risk factors such as age, alcohol consumption, and TP53 Arg72Pro polymorphisms might alter the effect of miR-34b/c rs4938723 polymorphism on cancer risk synergistically or antagonistically (Xu et al., 2011; Li et al., 2013; Zhang et al., 2014a; Pan et al., 2015). So, different combinations of multiple mechanisms adopted 
by different cancer types may contribute to the different effects of $\mathrm{miR}-34 \mathrm{~b} / \mathrm{c}$ rs4938723 polymorphism on them.

Sensitivity analysis also showed that omission of any single study did not have significant impact on the combined ORs. In addition, funnel plot did not reflect obvious asymmetry, and Egger's test further indicated no considerable publication bias under allelic model, homogeneous co-dominant model and recessive model comparison. The distribution of genotypes in the controls in all eligible studies did not deviate from HWE. Furthermore, all studies included in the meta-analysis were regarded as high quality according to the quality assessment criteria recommended by Xue et al (S1 Table 1 ). Therefore, the results were very robust and reliable.

To a certain extent, our meta-analysis still includes some limitations. First, the numbers for each type of digestive cancer were relatively small, so there is insufficient statistical power to investigate the association between mir-34b/c polymorphism and each type cancer; second, the lack of detailed original data of factor influencing the effect of miR-34b/c rs4938723 polymorphism on cancer risk, such as the age of the populations, alcohol consumption, methylation status of $\mathrm{mir}-34 \mathrm{~b} / \mathrm{c}$ gene CpG islands and TP53 Arg72Pro polymorphisms in the eligible studies may hinder our further analyses (Han et al., 2013; Li et al., 2013; Zhang et al., 2014a; Pan et al., 2015). Third, there were still not eligible studies to analyses miR-34b/c rs4938723 and digestive cancer risk in African \&Caucasian. Therefore, more studies are needed to explore the potential relationship between $\mathrm{miR}-34 \mathrm{~b} / \mathrm{c}$ rs4938723 polymorphisms and different types of digestive cancer susceptibility in ethnically diverse populations to consolidate our findings.

Taken together, our study provides evidence that $\mathrm{T}$ to $\mathrm{C}$ shift of the rs4938723 polymorphism reduce individual susceptibility to digestive cancer, Future studies with larger sample size and more ethnic groups on the association between miR-34b/c rs4938723 polymorphism and digestive cancers are required to confirm current findings.

\section{Acknowledgements}

We specially thank all authors contributed to the paper. And this study was partially supported by Guangzhou Medical science and technology project (No. 20141A010074)

\section{References}

Bensen JT, Tse CK, Nyante SJ, et al (2013). Association of germline microRNA SNPs in pre-miRNA flanking region and breast cancer risk and survival: the carolina breast cancer study. Cancer Causes Control, 24, 1099-109.

Chen X, Hu H, Guan X, et al (2012). CpG island methylation status of miRNAs in esophageal squamous cell carcinoma. Int J Cancer, 130, 1607-13.

Corney DC, Flesken-Nikitin A, Godwin AK, et al (2007). MicroRNA-34b and MicroRNA-34c are targets of p53 and cooperate in control of cell proliferation and adhesionindependent growth. Cancer Res, 67, 8433-8.
Corney DC, Hwang CI, Matoso A, et al (2010). Frequent downregulation of miR-34 family in human ovarian cancers. Clin Cancer Res, 16, 1119-28.

DerSimonian R, Laird N (1986). Meta-analysis in clinical trials. Control Clin Trials, 7, 177-88.

Egger M, Davey Smith G, Schneider M, et al (1997). Bias in meta-analysis detected by a simple, graphical test. $B M J$, 315, 629-34.

Gao LB, Li LJ, Pan XM, et al (2013). A genetic variant in the promoter region of $\mathrm{miR}-34 \mathrm{~b} / \mathrm{c}$ is associated with a reduced risk of colorectal cancer. Biol Chem, 394, 415-20.

Han Y, Pu R, Han X, et al (2013). Associations of pri-miR$34 \mathrm{~b} / \mathrm{c}$ and pre-miR-196a2 polymorphisms and their multiplicative interactions with hepatitis B virus mutations with hepatocellular carcinoma risk. PLoS One, 8, 58564.

He L, He X, Lim LP, et al (2007). A microRNA component of the p53 tumour suppressor network. Nature, 447, 1130-4.

Kalimutho M, Di Cecilia S, Del Vecchio Blanco G, et al (2011). Epigenetically silenced $\mathrm{miR}-34 \mathrm{~b} / \mathrm{c}$ as a novel faecal-based screening marker for colorectal cancer. Br J Cancer, 104, 1770-8.

Li L, Wu J, Sima X, et al (2013). Interactions of miR-34b/c and TP-53 polymorphisms on the risk of nasopharyngeal carcinoma. Tumour Biol, 34, 1919-23.

Lujambio A, Calin GA, Villanueva A, et al (2008). A microRNA DNA methylation signature for human cancer metastasis. Proc Natl Acad Sci U S A, 105, 13556-61.

Mantel N, Haenszel W (1959). Statistical aspects of the analysis of data from retrospective studies of disease. J Natl Cancer Inst, 22, 719-48.

Normand SL (1999). Meta-analysis: formulating, evaluating, combining, and reporting. Stat Med, 18, 321-59.

Oh J, Kim JW, Lee BE, et al (2014). Polymorphisms of the pri$\mathrm{miR}-34 \mathrm{~b} / \mathrm{c}$ promoter and TP53 codon 72 are associated with risk of colorectal cancer. Oncol Rep, 31, 995-1002.

Pan XM, Sun RF, Li ZH, et al (2015). Pri-miR-34b/c rs4938723 polymorphism is associated with a decreased risk of gastric cancer. Genet Test Mol Biomarkers.

Son MS, Jang MJ, Jeon YJ, et al (2013). Promoter polymorphisms of pri-miR-34b/c are associated with hepatocellular carcinoma. Gene, 524, 156-60.

Suzuki H, Yamamoto E, Nojima M, et al (2010). Methylationassociated silencing of microRNA-34b/c in gastric cancer and its involvement in an epigenetic field defect. Carcinogenesis, 31, 2066-73.

Suzuki R, Yamamoto E, Nojima M, et al (2014). Aberrant methylation of microRNA-34b/c is a predictive marker of metachronous gastric cancer risk. J Gastroenterol, 49, 1135-44.

Tao T, Chen S, Xu B, et al (2014). Association between hsa$\mathrm{miR}-34 \mathrm{~b} / \mathrm{c}$ rs4938723 $\mathrm{T}>\mathrm{C}$ promoter polymorphism and cancer risk: a meta-analysis based on 6,036 cases and 6,204 controls. Chin J Cancer Res, 26, 315-22.

Tian Q, Jia J, Ling S, et al (2014). A causal role for circulating miR-34b in osteosarcoma. Eur J Surg Oncol, 40, 67-72.

Toyota M, Suzuki H, Sasaki Y, et al (2008). Epigenetic silencing of microRNA-34b/c and B-cell translocation gene 4 is associated with $\mathrm{CpG}$ island methylation in colorectal cancer. Cancer Res, 68, 4123-32.

Vogt M, Munding J, Gruner M, et al (2011). Frequent concomitant inactivation of miR-34a and miR-34b/c by $\mathrm{CpG}$ methylation in colorectal, pancreatic, mammary, ovarian, urothelial, and renal cell carcinomas and soft tissue sarcomas. Virchows Arch, 458, 313-22.

Wang LQ, Kwong YL, Wong KF, et al (2014). Epigenetic inactivation of mir-34b/c in addition to mir-34a and DAPK 1 in chronic lymphocytic leukemia. J Transl Med, 12, 52. 
Tian-Xing Ji et al

Wang Z, Wu J, Zhang G, et al (2013). Associations of miR499 and miR-34b/c polymorphisms with susceptibility to hepatocellular carcinoma: an evidence-based evaluation. Gastroenterol Res Pract, 2013, 719202.

$\mathrm{Xu} \mathrm{Y,} \mathrm{Liu} \mathrm{L,} \mathrm{Liu} \mathrm{J,} \mathrm{et} \mathrm{al} \mathrm{(2011).} \mathrm{A} \mathrm{potentially} \mathrm{functional}$ polymorphism in the promoter region of $\mathrm{miR}-34 \mathrm{~b} / \mathrm{c}$ is associated with an increased risk for primary hepatocellular carcinoma. Int J Cancer, 128, 412-7.

Xue W, Zhu M, Wang Y, et al (2015). Association between PLCE1 rs2274223 A > G polymorphism and cancer risk: proof from a meta-analysis. Sci Rep, 5, 7986.

Yang C, Ma X, Liu D, et al (2014). Promoter polymorphisms of miR-34b/c are associated with risk of gastric cancer in a Chinese population. Tumour Biol, 35, 12545-54.

Yi DH, Wang BG, Zhong XP, et al (2014). Pri-miR-34b/c rs4938723 TC heterozygote is associated with increased cancer risks: evidence from published data. Tumour Biol, 35, 11967-75.

Yin J, Wang X, Zheng L, et al (2013). Hsa-miR-34b/c rs4938723 $\mathrm{T}>\mathrm{C}$ and hsa-miR-423 rs6505162 $\mathrm{C}>\mathrm{A}$ polymorphisms are associated with the risk of esophageal cancer in a Chinese population. PLoS One, $\mathbf{8}, 80570$.

Zhang J, Huang X, Xiao J, et al (2014a). Pri-miR-124 rs531564 and pri-miR-34b/c rs 4938723 polymorphisms are associated with decreased risk of esophageal squamous cell carcinoma in Chinese populations. PLoS One, 9, 100055.

Zhang S, Qian J, Cao Q, et al (2014b). A potentially functional polymorphism in the promoter region of $\mathrm{miR}-34 \mathrm{~b} / \mathrm{c}$ is associated with renal cell cancer risk in a Chinese population. Mutagenesis, 29, 149-54. 